\title{
A Pilot Study of Gemcitabine and Epirubicin Combination Chemotherapy as a Salvage Regimen for Recurrent Platinum Resistant and/or Refractory Epithelial Ovarian Cancer
}

\begin{abstract}
Abd El Halim Abu-Hamar
Clinical Oncology Department, Tanta University Hospital, Faculty of Medicine, Tanta University, Tanta, Egypt. Email: abuhamar65@hotmail.com,halim_am65@yahoo.com

Received June $21^{\text {st }}, 2011$; revised July $20^{\text {th }}, 2011$; accepted July $31^{\text {st }}, 2011$.

ABSTRACT

Background and Objectives: The objective of this study was to assess the antitumor activity and toxicity profile of gemcitabine combined with epirubicin in patients with recurrent platinum refractory ovarian epithelial cancer. Patients and Methods: Patients with recurrent platinum refractory ovarian cancer and with adequate hematologic, renal and hepatic function and an Eastern Cooperative Oncology Group (ECOG) performance status of 0 - 2 were enrolled. The regimen was Gemcitabine $1000 \mathrm{mg} / \mathrm{m}^{2}$ (day 1, 8) and Epirubicin $60 \mathrm{mg} / \mathrm{m}^{2}$ (day 1), the cycle was repeated at interval of 21 days. Results: Twenty eight patients were recruited and received 156 cycles of gemcitabine-epirubicin combination chemotherapy (median 6 cycles). Overall response rate was $42.9 \%$ (95\% CI equal 24.5 to 62.7) and tumor control rate was $75 \%$ (95\% CI equal 55.1 to 89.3). No complete responses were observed. Median progression-free and median overall survival times were 7 and 15 months, respectively. The most common grade 3/4 hematological toxicities were neutropenia (57.1\%), anemia (10.7\%), and thrombocytopenia (7.1\%), while the most common grade 3/4 non-hematological toxicities were mucositis (14.3\%) and vomiting (3.6\%). No treatment related deaths were observed. Conclusion: Gemcitabine combined with epirubicin regimen appeared to offer an acceptable clinical profile in patients with recurrent platinum-refractory epithelial ovarian cancer.
\end{abstract}

Keywords: Platinum-Refractory/Resistant Ovarian Cancer, Gemcitabine, Anthracyclines

\section{Introduction}

Ovarian cancer accounts for about 3\% of all cancers among women and also the leading cause of gynecologic cancer-related deaths. It ranks second among gynecologic cancers, following cancer of the uterine corpus. An estimated 21,880 new cases of ovarian cancer and 13,850 women are expected to die from ovarian cancer in The US in 2010 [1].

Treatment for advanced disease involves cytoreductive surgery followed by systemic treatment with paclitaxel and platinum. Overall tumor response rates associated with paclitaxel and platinum are relatively high and range from approximately $70 \%$ to $80 \%$. However, $50 \%$ to $75 \%$ of responders will relapse within approximately 18 months after completing first-line therapy and require further systemic therapy $[2,3]$.

Predictors for recurrence include late stage, residual disease, advanced age, histologic grade, poor performance status, clear cell or mucinous histology, and suboptimal normalization of CA125 levels following first-line therapy $[4,5]$.

Only a small proportion of recurrent ovarian cancer patients may benefit from surgical treatment of recurrence [6], since in most cases ovarian cancer patients recur with diffuse carcinomatosis or multiple nodule abdominal disease [7], and require medical treatment. The choice of the drug or drug combinations to be used at recurrence is conditioned by the duration of platinumfree interval (PFI). Patients recurring within 6 months from completion of primary chemotherapy are considered platinum resistant and are usually treated with salvage non platinum chemotherapy, while cases with a PFI $>6$ months are considered platinum sensitive and exhibit rates of response to platinum-based rechallenge, ranging 
from $27 \%$ to $59 \%$ according to the duration of PFI [8$10]$.

Platinum-resistant patients present a very poor prognosis with short-lasting response and survival. Several single chemotherapeutic agents have been used in this setting and have demonstrated modest activity such as topotecan [11,12], gemcitabine [13,14], liposomal doxorubicin [15], vinorelbine [16], oral etoposide [17,18], and ifosfamide [19]. Epirubicin, a Doxirubocin analogue, is active agent in first and second-line treatment of epithelial ovarian cancer [20].

In this subset of patients the response rate reported for different treatments is quite similar, varying from $14 \%$ to $34 \%$ and, at present, no drug may be considered the treatment of choice. The principal end-point of salvage treatment in this subset of patients is represented by the palliation of symptoms and the maintenance of acceptable quality of life [21]. All present new drugs or combinations, potentially active and with an acceptable toxicity profile, are continuously evaluated in this subset of patients in order to convert a higher response rate in a longer survival [22].

Many reasons could account for combination of gemcitabine and anthracyclines including proven activity in ovarian cancer [20,23-25], synergistic anti-proliferative effect in vitro and in vivo trials, absence of any crossresistance as their mechanism of action are different, in addition, absence of any overlapping toxicity profiles [25, 26].

We conducted this prospective pilot, single institution study to evaluate the efficacy and toxicity of the combination of Gemcitabine and Epirubicin in treatment patients aged greater than 18 years and less than 75 years, with recurrent platinum-refractory epithelial ovarian cancer.

\section{Materials and Methods}

\subsection{Patient Selection}

Eligible patients were required to have histologically confirmed, recurrent epithelial ovarian cancer that was resistant and/or refractory to platinum based chemotherapy (progression during platinum therapy or in the following 6 months). Prior chemotherapy was allowed in conjunction with primary surgery 6 months prior to study entry. All patients were required to have measurable recurrent lesions, age less than 75 years and greater than 18 years, World Health Organization performance status of 0 to 2 , adequate cardiac function ( $\mathrm{EF}>60 \%$ ), adequate bone marrow reserve, adequate renal and hepatic functions.

Exclusion criteria included the following: the presence of symptomatic brain metastases; a history of ventricular arrhythmia, congestive heart failure, or documented myocardial infarction and uncontrolled severe infection which would limit full compliance with the study or would expose the patient to extreme risk.

\subsection{Investigations}

The following parameters were assessed at baseline: $\mathrm{CA}_{125}$ level, WHO performance status, weight, pelvic examination, abdomino-pelvic computed tomography (CT) scan, chest $\mathrm{x}$-ray, ECG, echocardiography, isotopic bone scan (if indicated), blood counts (hemoglobin, granulocytes, and platelets) and blood chemistry (renal and liver function tests). $\mathrm{CA}_{125}$ level, performance status, weight, pelvic and abdominal examination were performed before each cycle together with an assessment of toxicity according to the National Cancer Institute-Common Toxicity Criteria (NCI-CTCAE ver. 3.0). Scans were planned every 3 cycles or when progressive disease was suspected on clinical examination. Blood counts were performed weekly.

\subsection{Treatment}

Gemcitabine was given by intravenous infusion at 1000 $\mathrm{mg} / \mathrm{m}^{2}$ over $1 / 2$ hour (Day 1 and 8 ), and Epirubicin was given by single intravenous infusion at $60 \mathrm{mg} / \mathrm{m}^{2}$ (Day 1). Treatment was repeated every 3 weeks and continued for 6 cycles unless there was evidence of disease progression or unacceptable toxicity. Patients showing objective response after 6 cycles continued to a maximum of 9 cycles. Patients were pre-medicated with $8 \mathrm{mg}$ ondansetron, $2 \mathrm{ml}(8 \mathrm{mg})$ of dexamethasone, $50 \mathrm{mg}$ of diphenhydramine, and $50 \mathrm{mg}$ of ranitidine given intravenously. The protocol provided for a decrease in Gemcitabine and Epirubicin dose in patients experiencing grade 4 hematological toxicity or grade 3 non-hematological toxicities. G-CSF support was allowed in case of prolonged leucopenia ( $>7$ days) or febrile neutropenia in the prior cycle.

\subsection{Treatment Response and Patient Evaluation}

Tumor response assessments were performed after 3 and 6 cycles. Response to treatment was assessed using WHO guidelines [27]. Evaluation was done using computed tomography (CT) owing to its convenient diagnosis of target lesion progress and identification of emerging new lesions. Assessment of $\mathrm{CA}_{125}$ level was repeated 1 month after the patient stopped therapy and then every 3 months together with assessments of blood counts, chemistry, weight, performance status, toxicity, and pelvic examination. An increase in $\mathrm{CA}_{125}$ levels not associated with radiologic or clinical evidence of tumor progression was not used as the sole indicator of progressive 
disease. Follow-up visits were scheduled every 3 months in the first 2 years after cessation of treatment and every 6 months thereafter.

Toxicity Evaluation: Toxicities were graded according to the National Cancer Institute-Common Toxicity Criteria (NCI-CTCAE ver. 3.0). Treatment period was defined as the period from the initiation of therapy to 3 weeks after the last day of administration of Gemcitabine and Epirubicin.

\subsection{Statistical Analysis}

The SPSS 10 statistics program was used for statistical analysis. The primary endpoints of this study were tumor response, overall survival time and time to disease progression. The secondary end point was to assess the safety and toxicity profile of this regimen. Overall survival was measured from the first day of study treatment until death or last follow-up. Patient's characteristics were summarized by descriptive statistics (median, range and frequency).The percentage of patients experiencing clinical response (PR) was reported along with the corresponding exact 95\% confidence intervals (CI). [28] Kaplan-Meier curves [29] (with 95\% CI) were plotted for OS.

\section{Results}

\subsection{Patients Characteristics}

A total of 28 consecutive platinum-resistant or refractory recurrent ovarian carcinoma patients were treated at Clinical Oncology Department, Faculty of Medicine, Tanta University, Egypt and enrolled in this phase II trial from January 2007 to December 2010.

Baseline characteristics of patients with recurrent platinum resistant ovarian epithelial cancer are listed in Table 1. The median age of study participants was 57 years (range, $42-71$ years, and $\mathrm{SD} \pm 7.11$ ). All patients had previously received platinum-based chemotherapy treatment. All patients had relapse or progression to previous treatment within 6 months. Sixteen patients (57.1\%) presented with WHO performance status of 2. At initial diagnosis, all patients had Stage III-IV disease $(67.9 \%$ and $32.1 \%$ respectively), according to The FIGO staging system. Histology was, serous cystadencarcinoma in 11 patients, mucinous cystadencarcinoma in 8 , endometroid adenocarcinoma in 5 and undifferentiated carcinoma in 4 patients.

\subsection{Treatment}

In total, 156 cycles of Gemcitabine and Epirubicin were administered (median, 6 cycles; range, 3 - 9 cycles). All cycles were given at the initial planned doses, and no
Table 1. Baseline characteristics of patients with recurrent platinum resistant ovarian epithelial cancer.

No. (28) \%

\author{
Age in years \\ Range 42 - 71 \\ Mean 56.2 \\ Median 57 \\ Std. Deviation \pm 7.11
}

$\begin{array}{lcc}\text { CA } 125 \text { level } & & \\ \text { Range } 20.0 \text { - } 4459.0 \mathrm{IU} / \mathrm{ml}) & & \\ \text { Mean } 963.5 \mathrm{IU} / \mathrm{ml} & & \\ \text { Median } 240 \mathrm{IU} / \mathrm{ml} & & \\ & & \\ \text { Performance status } & 12 & 42.9 \\ \text { I } & 16 & 57.1 \\ \text { II } & & \\ & & \\ \text { Pathological type } & 11 & 39.3 \\ \text { Serous cystadencarcinoma } & 8 & 28.6 \\ \text { Mucinous cystadencarcinoma } & 5 & 17.8 \\ \text { Endometroid Adenocarcinoma } & 4 & 14.3 \\ \text { Undifferentiated ca } & & \\ & & \\ \text { Tumor grade } & 9 & 32.1 \\ \text { Grade II } & 16 & 57.1 \\ \text { Grade III } & 3 & 10.7 \\ \text { Unknown } & & \\ & & \\ \text { Initial Tumor Stage } & 19 & 67.9 \\ \text { Stage III } & 9 & 32.1 \\ \text { Stage IV } & & \\ \text { Site of recurrence } & & \\ \text { Peritoneum } & 15 & 53.6 \\ \text { Liver } & 4 & 14.3 \\ \text { Pelvic \&/or para-aortic LNs } & 2 & 7.1 \\ \text { Lung } & 2 & 7.1 \\ \text { Mixed } & 5 & 17.8 \\ & & \\ & & \end{array}$

LNs: lymph nodes.

treatment delays because of toxicity were reported.

\subsubsection{Response to Treatment}

The overall response rate was $42.9 \%$ (with $95 \% \mathrm{CI}$ equal 24.5 - 62.7), and 12 patients showed partial responses. No complete responses were observed. Stable disease was recorded in 9 patients $(32.1 \%$, with $95 \%$ CI equal 15.8 - 52.3), and progressive disease was recorded in 7 patients (25\% with 95\% CI equal 10.7 - 44.9) (Table 2). Patients failed on the studied regimen, (7 patients) received best supportive care and further treatment was at the physician's discretion.

\subsubsection{Survival}

The median follow up was 12 months (range $4-22$ 
Table 2. Best overall tumor response by WHO response criteria (primary endpoint).

\begin{tabular}{lccc}
\hline Response & No & \% & 95\% Confidence Interval \\
\hline CR & 0 & 0.0 & 0.00 \\
PR & 12 & 42.9 & $24.5-62.7$ \\
SD & 9 & 32.1 & $15.8-52.3$ \\
Progressive disease & 7 & 25 & $10.7-44.9$ \\
$\begin{array}{l}\text { Overall tumor } \\
\text { response (PR + SD) }\end{array}$ & 21 & 75 & $55.1-89.3$ \\
\hline
\end{tabular}

months). Nine patients were censored at the time of analysis. The median time to progression was 7 months (95\% confidence interval, 6.1 - 7.9; SE: 0.46 ). The median overall survival was 15 months $(95 \%$ confidence interval, 11.1 - 18.9; SE: 2.01) (Table 3), and the 1-year overall survival rate was $57.1 \%$ (Figure 1).

\subsubsection{Toxicity}

Toxicity assessment of the 28 patients who received treatment is detailed in Table 4. Grade 3 - 4 neutropenia and anemia were reported in $57.1 \%$ and $10.7 \%$ of patients, respectively. The most frequent Grade 3 - 4 non-

Table 3. Overall survival and progression-free survival of all patients $(\mathbf{n}=\mathbf{2 8})$.

\begin{tabular}{cccc}
\hline Secondary end points & & $\begin{array}{c}\text { 95\% confidence } \\
\text { Interval }\end{array}$ & $\begin{array}{c}\text { Standard } \\
\text { Error }\end{array}$ \\
\hline $\begin{array}{c}\text { Overall survival (months) } \\
\text { Range }\end{array}$ & $4-24$ & & \\
Median & 15 & $11.1-18.9$ & 2.01 \\
Mean & 13.9 & $11.9-15.9$ & 1.03 \\
Time to DP (months) & & & \\
Range & $3-10$ & & 0.46 \\
Median & 7 & $6.1-7.9$ & 0.36 \\
Mean & 6.78 & $6.1-7.5$ & \\
\hline
\end{tabular}

DP: disease progression.

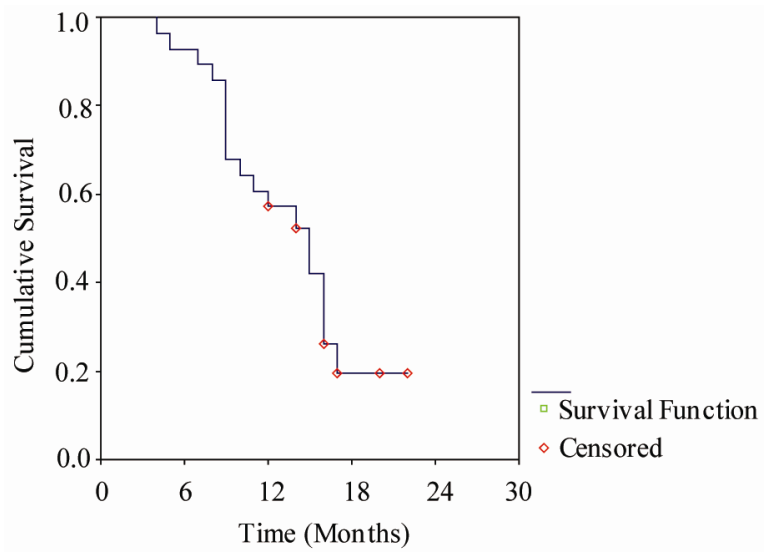

Figure 1. Overall survival of all patients.
Table 4. Treatment-related adverse events.

\begin{tabular}{lcccc}
\hline \multirow{2}{*}{ Type of toxicity } & \multicolumn{3}{c}{ Any grade } & Grade $\mathbf{3}-\mathbf{4}$ \\
\cline { 2 - 5 } & No & $\%$ & No & $\%$ \\
\hline Hematological & 22 & 78.6 & 16 & 57.1 \\
Neutropenia & 17 & 60.7 & 3 & 10.7 \\
Anemia & 15 & 53.6 & 2 & 7.1 \\
Thrombocytopenia & 23 & 82.1 & 0 & 0.0 \\
Alopecia & 20 & 71.4 & 4 & 14.3 \\
Mucositis & 21 & 75 & 1 & 3.6 \\
Nausea and/or vomiting & 9 & 32.1 & 0 & 0.0 \\
Tranaminases & 6 & 21.4 & 0 & 0.0 \\
Constipation & 6 & 21.4 & 0 & 0.0 \\
Diarrhea & 2 & 7.1 & 0 & 0.0 \\
Infection & & & &
\end{tabular}

hematologic toxicities observed were mucositis (14.3\%), and vomiting (3.6\%). No patients discontinued therapy because of toxicity. There were no treatment-related deaths during the study.

\section{Discussion}

In this study, gemcitabine combined with epirubicin in patients with recurrent platinum refractory ovarian epithelial cancer appeared to have good clinical efficacy and an acceptable safety profile. It was associated with a $42.9 \%$ overall response rate and a median overall survival of 15 months.

Platinum with concurrent taxane-based chemotherapy is the non-surgical standard of care for locally advanced ovarian epithelial cancer patients, and is also considered the standard adjuvant therapy for high-risk post-operative patients [30-32]. Although the initial response rate to chemotherapy was high, some patients experience relapse quickly and were not good candidates for re-treatment with these agents. Therefore, identification of active agents in patients with platinum refractory disease is important. Ideally, second-line agents should lack cross resistance to previous agents. Because of the palliative nature of second-line treatment, these agents should also have a favorable toxicity profile [33].

There have been several trials to improve survival of patients with platinum-refractory ovarian epithelial cancer. Lund et al. [34], and Shapiro et al. [35] reported the results of gemcitabine and found that response rate was $15 \%$ to $28 \%$ in patients with documented progressive disease or recurrence. Vermoken et al., [20] showed that epirubicin (E), a doxirubicin analogue is active in first and second-line treatment of ovarian epithelial cancer.

Many reasons could account for combination of gemcitabine and anthracyclines including proven activity in ovarian cancer [20,23-25], synergistic anti-proliferative 
effect in vitro and in vivo trials, absence of any crossresistance as their mechanism of action are different, in addition, absence of any overlapping toxicity profiles $[25$, 26].

Chow et al., [36] reported an overall response rate for gemcitabine combined with epirubicin $\left(1000 \mathrm{mg} / \mathrm{m}^{2}\right.$ and $60 \mathrm{mg} / \mathrm{m}^{2}$ respectively) in 13 recurrent, platinum-resistant ovarian epithelial cancer patients of $23 \%$. In a different phase I-II trial on platinum-resistant ovarian epithelial cancer patients, Goff et al., [37] reported a median overall survival of 12 months, and an overall response rate of $24 \%$ (11/49), with the most frequent side-effect being hematological, and Quality of life evaluation indicated a good tolerance of this regimen.

Based on the previous results, this phase II trial was conducted to evaluate the efficacy and toxicity of gemcitabine combined with epirubicin as a $2^{\text {nd }}$ line chemotherapy in patients with recurrent platinum resistant and/ or refractory ovarian epithelial cancer.

The primary end points of this study were to assess the efficacy of this regimen: Overall response rates, time to disease progression (TTP), and overall survival (OS). The overall response was $75 \%$. These results compare favorably with those reported by Murgia et al. [38] and Chu et al. [39] who used the same regimen in treatment of patients with platinum-resistant ovarian cancer. Murgia et al. [38] who treated 48 patients reported that the overall tumor response rate was $76.7 \%$ (CR $2.1 \%$, PR 41.7\% and SD 33.3\%), while Chu et al. [39] who treated 60 patients with the same regimen plus ifosfamide reported that the overall tumor response rate was $76.7 \%$ (PR 36.7\% and SD 38.3\%). On the other hand, our results are lower than those reported by Galligioni et al. [40] in a phase II Italian study which included 27 patients with platinum-resistant ovarian cancer, the overall tumor response rate was $81.4 \%$ (CR $3.7 \%$, PR $44.4 \%$ and SD $33.3 \%$ ). This could be explained by the fact that all patients in our study had recurrent platinum resistant and/or refractory disease (PFI $<6$ months), while in Galligioni et al. [40] study 6 patients out of 27 had PFI $\geq 6<12$ months. Our results are higher than those reported by Goff et al. [37] They reported that the overall response rate was $24 \%$. This could be explained by the fact that, the chemotherapy in this study was given as a first line salvage treatment while in Goff et al. study [37], the median number of previous chemotherapy regimens for the all patients was 2 (range $1-5$ ). Also in Goff et al. study [37], they used a different dose schedule in (gemcitabine $700 \mathrm{mg} / \mathrm{m}^{2}$ on days $1 \& 8$, and doxorubicin $20 \mathrm{mg} / \mathrm{m}^{2}$ on days 1 and 8 ).

In the present study the median time to disease progression and the median overall survival time were 7 and
15 months respectively. While, Galligioni et al. [40] had reported a median time to disease progression of 8 months, Murgia et al. [38] reported a median progression-free and overall survival of 7 and 23.5 months, respectively, and, Chu et al. [39] reported a median time to disease progression and a median overall survival time of 7 and 20 months respectively.

The secondary end point was to assess the safety and toxicity profile of this regimen. Most of the adverse events observed in this study were predictable and manageable. The incidence of grade $3 / 4$ neutropenia was $57.1 \%$, and only 3 of 28 patients (10.7\%) had developed febrile neutropenia. Adverse events required dose reduction in only 10 of 156 cycles $(6.4 \%)$. The usage of GCSF occurred in 35 of 156 cycles $(22.4 \%)$. The other Grade 3 - 4 hematologic toxicities were anemia $(10.7 \%)$, and thrombocytopenia (7.1\%). The most frequent Grade 3 - 4 non-hematologic toxicities observed were mucositis $(14.3 \%)$, and vomiting (3.6\%). No patients discontinued therapy because of toxicity. There were no treatmentrelated deaths during this study. These results are nearly consistant with those reported by Murgia et al. [38] who reported that long-term adverse effects associated with $\mathrm{G} / \mathrm{E}$ treatment include beside alopecia in all pts, major grade 3 - 4 toxicities consisted of, anemia $4 \%$, neutropenia $56 \%$, thrombocytopenia $6 \%$, vomiting $4 \%$, diarrhoea $2 \%$, hepatotoxicity $8 \%$, and mucositis $6 \%$. While Galligioni et al. [40] reported that Grade 3 - 4 toxicities consisted of neutropenia (58\%), thrombocytopenia (3\%), anemia (10\%), hepatotoxicity (13\%), and mucositis (7\%). On the other hand, Chu et al. [39] reported that Grade 3 4 toxicities consisted of neutropenia (31\%), while, digestive reactions were only of grade $1-2$, and accounted for $42 \%$.

In conclusion, the current results suggest that, the regimen of gemcitabine combined with epirubicin appeared to be an active and safe chemotherapy regimen for patients with recurrent platinum-refractory epithelial ovarian cancer. Further prospective investigation of this regimen to optimize doses and scheduling is necessary.

\section{REFERENCES}

[1] A. Jemal, R. Siegel, E.Ward, et al., "Cancer Statistics," CA: A Cancer Journal for Clinicians, Vol. 59, No. 4, 2009, pp. 225-249. doi:10.3322/caac.20006

[2] A. du Bois, M. Quinn, T. Thigpen, J. Vermorken, E. VallLundqvist, M. Bookman, D. Bowtell, M. Brady, A. Casado, A. Cervantes, et al., "2004 Consensus Statements on the Management of Ovarian Cancer: Final Document of the 3rd International Gynecologic Cancer Intergroup Ovarian Cancer Consensus Conference (GCIG OCCC 2004)," Annals of Oncology, Vol. 16, No. 8, 2005, pp. viii7-viii12. 
[3] R. F. Ozols, B. N. Bundy, B. E. Greer, et al., "Phase III Trial of Carboplatin and Paclitaxel Compared with Cisplatin and Paclitaxel in Patients with Optimally Resected Stage III Ovarian Cancer: A Gynecologic Oncology Group Study," Journal of Clinical Oncology, Vol. 21, No. 17, 2003, pp. 3194-200. doi:10.1200/JCO.2003.02.153

[4] A. Gadducci, F. Landoni, T. Maggino, et al., "Serum CA125 Assay at the Time of Relapse Has No Prognostic Relevance in Patients Undergoing Chemotherapy for Recurrent Ovarian Cancer: A Multicenter Italian Study," International Journal of Gynecological Cancer, Vol. 7, No. 1, 1997, pp. 78-83. doi:10.1046/j.1525-1438.1997.00424.x

[5] A. P. Heintz, F. Odicino, P. Maisonneuve, et al., "Carcinoma of the Ovary. FIGO 6th Annual Report on the Results of Treatment in Gynecological Cancer," International Journal of Gynecology \& Obstetrics, Vol. 95, No. 1, 2006, pp. S161-S192. doi:10.1016/S0020-7292(06)60033-7

[6] P. Harter and A. du Bois, "The Role of Surgery in Ovarian Cancer with Special Emphasis on Cytoreductive Surgery for Recurrence," Current Opinion in Oncology, Vol. 17, No. 5, 2005, pp. 505-514. doi:10.1097/01.cco.0000174166.06734.c7

[7] G. Ferrandina, F. Legge, V. Salutari, et al., "Impact of Pattern of Recurrence on Clinical Outcome of Ovarian Cancer Patients: Clinical Considerations," European Journal of Cancer, Vol. 42, No. 14, 2006, pp. 2296-2302. doi:10.1016/j.ejca.2006.03.025

[8] T. J. Herzog, "The Current Treatment of Recurrent Ovarian Cancer," Current Oncology Reports, Vol. 8, No. 6, 2006, pp. 448-454. doi:10.1007/s11912-006-0074-9

[9] M. Markman, "Recurrence within 6 Months of Platinum Therapy: An Adequate Definition of "Platinum-Refractory" Ovarian Cancer?" Gynecologic Oncology, Vol. 69, No. 2, 1998, pp. 91-92. doi:10.1006/gyno.1998.4997

[10] M. Markman, R. Rothman, T. Hakes, et al., "Second-Line Platinum Therapy in Patients with Ovarian Cancer PreviOusly Treated with Cisplatin," Journal of Clinical Oncology, Vol. 9, No. 3, 1991, pp. 389-393.

[11] M. A. Bookman, H. Malmstrom, G. Bolis, A. Gordon, A. Lissoni, J. B. Krebs and S. Z. Fields, "Topotecan for the Treatment of Advanced Epithelial Ovarian Cancer: An Open-Label Phase II Study in Patients Treated after Prior Chemotherapy That Contained Cisplatin or Carboplatin and Paclitaxel," Journal of Clinical Oncology, Vol. 16, No. 10, 1998, pp. 3345-3352.

[12] G. J. Creemers, G. Bolis, M. Gore, G. Scarfone, A. J. Lacave, J. P. Guastalla, et al., "Topotecan, an Active Drug in the Second-Line Treatment of Epithelial Ovarian Cancer: Results of a Large European Phase II Study," Journal of Clinical Oncology, Vol. 14, No. 12, 1996, pp. 3056-3061.

[13] J. D. Shapiro, M. J. Millward, D. Rischin, M. Michael, V. Walcher, P. A. Francis, et al., "Activity of Gemcitabine in Patients with Advanced Ovarian Cancer: Responses Seen Following Platinum and Paclitaxel," Gynecologic Oncol- ogy, Vol. 63, No. 1, 1996, pp. 89-93. doi:10.1006/gyno.1996.0284

[14] B. Lund, O. P. Hansen, K. Theilade, M. Hansen and J. P. Neijt, 'Phase II Study of Gemcitabine (2',2'-Difluorodeoxycytidine) in Previously Treated Ovarian Cancer Patients," Journal of the National Cancer Institute, Vol. 86, No. 20, 1994, pp. 1530-1533. doi:10.1093/jnci/86.20.1530

[15] F. M. Muggia, J. D. Hainsworth, S. Jeffers, P. Miller, S. Groshen, M. Tan, et al., "Phase II Study of Liposomal Doxorubicin in Refractory Ovarian Cancer: Antitumor Activity and Toxicity Modification by Liposomal Encapsulation," Journal of Clinical Oncology, Vol. 15, No. 3, 1997, pp. 987-993.

[16] E. Bajetta, A. Di Leo, L. Biganzoli, L. Mariani, F. Cappuzzo, M. Di Bartolomeo, et al., "Phase II Study of Vinorelbine in Patients with Pretreated Advanced Ovarian Cancer: Activity in Platinum-Resistant Disease," Journal of Clinical Oncology Vol. 14, No. 9, 1996, pp. 25462551.

[17] P. J. Hoskins and K. D. Swenerton, "Oral Etoposide Is Active against Platinum Resistant Epithelial Ovarian Cancer," Journal of Clinical Oncology, Vol. 12, No. 1, 1997, pp. 60-63.

[18] P. G. Rose, J. A. Blessing, A. R. Mayer and H. D. Homesley, "Prolonged Oral Etoposide as Second-Line Therapy for Platinum-Resistant and Platinum-Sensitive Ovarian Carcinoma: A Gynecologic Oncology Group Study," Journal of Clinical Oncology, Vol. 16, No. 2, 1998, pp. 405-410.

[19] G. P. Sutton, J. A. Blessing, H. D. Homesley, M. L. Berman and J. Malfetano, "Phase II Trial of Ifosfamide and Mesna in Advanced Ovarian Carcinoma: A Gynecologic Oncology Group Study," Journal of Clinical Oncology, Vol. 7, No. 11, 1989, pp. 1672-1676.

[20] J. B. Vermorken, A. Kobierska, M. E. van der Burg, B. Chevallier, F. Zanaboni, W. W. ten Bokkel Huinink, et al., "High-Dose Epirubicin in Platinum-Pretreated Patients with Ovarian Carcinoma: The EORTC-GCCG Experience," European Journal of Gynaecological Oncology, Vol. 16, No. 6, 1995, pp. 433-438.

[21] D. S. Alberts, "Treatment of Refractory and Recurrent Ovarian Cancer," Seminars in Oncology, Vol. 1, No. 1, 1999, pp. 8-14.

[22] M. Harries and M. Gore, "Chemotherapy for Epithelial Ovarian Cancer-Treatment of Recurrent Disease," Lancet Oncology, Vol. 3, No. 9, 2002, pp. 537-545. doi:10.1016/S1470-2045(02)00847-1

[23] M. Markman, K. Webster, K. Zanotti, B. Kulp, G. Peterson and J. Belinson, "Phase II Trial of Single Agent Gemcitabine in Platinum-Paclitaxel Resistant Ovarian Cancer," Gynecologic Oncology, Vol. 90, No. 3, 2003, pp. 593-596. doi:10.1016/S0090-8258(03)00399-8

[24] A. N. Gordon, C. O. Granai, P. G. Rose, J. Hainsworth, A. Lopez, C. Weissman, et al., "Phase II Study of Liposomal Doxorubicin in Platinum- and Paclitaxel-Refractory Epithelial Ovarian Cancer," Journal of Clinical Oncology, Vol. 
18, No. 7, 2000, pp. 3093-3100.

[25] A. N. Gordon, J. T. Fleagle, D. Guthrie, D. E. Parkin, M. E. Gore and A. J. Lacave, "Recurrent Epithelial Ovarian Carcinoma: A Randomized Phase III Study of Pegylated Liposomal Doxorubicin versus Topotecan," Journal of Clinical Oncology, Vol. 19, No. 14, 2001, pp. 3312-3322.

[26] W. Zoli, L. Ricotti, F. Barzanti, M. Dal Susino, G. L. Frassineti, C. Milandri, et al., "Schedule-Dependent Interaction of Doxorubicin, Paclitaxel and Gemcitabine in $\mathrm{Hu}-$ man Breast Cancer Cell Lines," International Journal of Cancer, Vol. 80, No. 3, 1999, pp. 413-416.

[27] A. B. Miller, B. Hoogstraten, M. Staquet and A. Winkler, "Reporting Results of Cancer Treatment," Cancer, Vol. 47, No. 1, 1981, pp. 207-214.

[28] P. Armitage, G. Berry and J. N. S. Mathews, "Statistical Methods in Medical Research," 4th Edition, Blackwell Sciences Ltd, Oxford, 2002. doi:10.1002/9780470773666

[29] E. Kaplan and P. Meier, "Nonparametric Estimation from Incomplete Observations," Journal of the American Statistical Association, Vol. 53, 1958, pp. 457-481. doi: $10.2307 / 2281868$

[30] P. Zola and A. Ferrero, "Is Carboplatin-Paclitaxel Combination the Standard Treatment of Elderly Ovarian Cancer Patients?" Annals of Oncology, Vol. 18, No. 2, 2007, pp. 213-214. doi:10.1093/annonc/mdl483

[31] M. Harries and M. Gore, "Chemotherapy for Epithelial Ovarian Cancer-Treatment at First Diagnosis," Lancet Oncology, Vol. 3, No. 9, 2002, pp. 529-536.

[32] A. Du Bois, H. J. Luck, T. Bauknecht, V. Mobus, H. Bochtler, K. Diergarten, et al., "Phase I/II Study of the Combination of Carboplatin and Paclitaxel as First-Line Chemotherapy in Patients with Advanced Epithelial Ovarian Cancer," Annals of Oncology, Vol. 8, No. 4, 1997, pp. 355-361. doi:10.1023/A:1008267419453

[33] D. G. Mutch, M. Orlando, T. Goss, M. G. Teneriello, A. N. Gordon, S. D. McMeekin, et al., "Randomized Phase III Trial of Gemcitabine Compared with Pegylated Liposomal Doxorubicin in Patients with Platinum-Resistant Ovarian Cancer," Journal of Clinical Oncology, Vol. 25, No. 19, 2007, pp. 2811-2818.
[34] B. Lund, O. P. Hansen, K. Theilade, M. Hansen and J. P. Neijt, "Phase II Study of Gemcitabine in Previously Treated Ovarian Cancer Patients," Journal of the National Cancer Institute, Vol. 86, No. 20, 1994, pp. 1530-1533. doi:10.1093/jnci/86.20.1530

[35] J. D. Shapiro, M. J. Millward, D. Rishin, M. Michael, V. Walcher, P. A. Francis and G. C. Toner, "Activity of Gemcitabine in Patients with Advanced Ovarian Cancer: Responses Seen Following Platinum and Paclitaxel," Gynecologic Oncology, Vol. 63, No. 1, 1996, pp. 89-93. doi:10.1006/gyno.1996.0284

[36] K. U. Chow, J. Ries, E. Weidmann, F. Pourebrahim, S. Napieralski, M. Stieler, et al., "Induction of Apoptosis Using 2',2'-Difluorodeoxycytidine (Gemcitabine) in Combination with Antimetabolites or Antracyclines on Malignant Lymphatic and Myeloid Cells. Antagonism or Symergism Depends on Incubation Schedule and Origin of Neoplastic Cells," Annals of Hematology, Vol. 79, No. 9, 2000, pp. 485-492. doi:10.1007/s002770000181

[37] B. A. Goff, T. Thompson, B. E. Greer, A. Jacobs and B. Storere, "Treatment of Recurrent Platinum Resistant Ovarian or Peritoneal Cancer with Gemcitabine and Doxorubicin: A Phase I/II Trial of the Puget Sound Oncology Consortium (PSOC 1602)," American Journal of Obstetrics and Gynecology, Vol. 188, No. 6, 2003, pp. 15561564. doi: $10.1067 / \mathrm{mob} .2003 .398$

[38] V. Murgia, O. Caffo, R. Sorio, C. Griso, A. Scalone, A. Ferro, et al., "Gemcitabine (G) and Epirubicin (E) Combination, in Platinum-Resistant or Refractory Advanced Ovarian Cancer (PROC) Patients: Results of a Multicentric Phase II Trial," Journal of Clinical Oncology, Vol. 26, No. 15, 2008, p. 5566.

[39] P. Chu, C. H. Lu, X. G. Sheng, C. X. Zhou, D. P. Li, X. L. DU and N. F. Liu, "Gemcitabine Based Combination Chemotherapy, a New Salvage Regimen for Recurrent Platinum Resistant Epithelial Ovarian Cancer," Zhonghua Fu Chan Ke Za Zhi, Vol. 45, No. 11 2010, pp. 808-812.

[40] E. Galligioni, C. Arcuri, R. Sorio and C. Griso, "Gemcitabine and Anthracyclines in Platinum-Resistant Ovarian Cancer," Annals of Oncology, Vol. 17, No. 5, 2006, pp. 195-198. 